\title{
Enquête
}

Archives de la revue Enquête

4 | 1996

La ville des sciences sociales

\section{La ville à travers ses mots}

The City through its words

Jean-Charles Depaule et Christian Topalov

\section{OpenEdition}

\section{Journals}

Édition électronique

URL : http://journals.openedition.org/enquete/963

DOI : 10.4000/enquete.963

ISSN : 1953-809X

\section{Éditeur :}

Cercom, Éditions Parenthèses

Édition imprimée

Date de publication : 1 novembre 1996

Pagination : 247-266

\section{Référence électronique}

Jean-Charles Depaule et Christian Topalov, «La ville à travers ses mots », Enquête [En ligne], 4| 1996, mis en ligne le 12 juillet 2013, consulté le 19 avril 2019. URL : http://journals.openedition.org/ enquete/963; DOI : 10.4000/enquete.963

Ce document a été généré automatiquement le 19 avril 2019. 


\title{
La ville à travers ses mots
}

\author{
The City through its words
}

Jean-Charles Depaule et Christian Topalov

\begin{abstract}
$1 \quad$ Approcher les villes par les mots qui les désignent et en désignent les différentes parties? Depuis fort longtemps, voyageurs et ethnologues, géographes et historiens, lexicographes et linguistes ont recueilli des «mots de la ville». Des trésors ont été ainsi rassemblés, le plus souvent de manière dispersée et en marge d'autres préoccupations. Il est rare qu'ils soient mis à profit autrement que pour dresser des listes assorties de définitions. L'observateur parfois s'attache à la langue, sa richesse, son évolution, ses particularités et guère aux objets du discours. Parfois, à l'inverse, l'attention porte sur ceux-ci et l'on a tôt fait de postuler que les mots désignent simplement des choses qui sont là avant d'être nommées. Il est possible, cependant, de regarder les systèmes lexicaux comme des traces et des vecteurs de dynamiques sociales qui s'inscrivent à la fois dans la ville et dans le langage. Les réflexions que nous présentons ici prennent appui sur un programme de recherche, « Les mots de la ville », qui s'aventure sur ce chemin et dont elles développent les principales orientations ${ }^{1}$.
\end{abstract}

\section{Un état des lieux}

2 L'idée, tout hugolienne, que la ville est un livre ouvert et son espace une sorte de langage, ou d'écriture, est familière. Roland Barthes l'avait ravivée à la fin des années soixante, mais son propos était sans doute trop général pour constituer un véritable programme de travail. Se définissant lui-même comme un amateur, «amateur de signes, celui qui aime les signes, amateur de villes, celui qui aime la ville », il insistait notamment sur l'intérêt d'en multiplier les lectures'2.

De nombreuses recherches, parfois inspirées de Greimas ou Umberto Eco, qui considèrent l'espace comme un langage non verbal, après une période d'engouement, sont tombées dans l'oubli, voire la dépréciation. Cela ne doit pas en occulter l'intérêt, au moins méthodologique ${ }^{3}$. L'échelle qu'elles ont retenue est plutôt celle des bâtiments, dont elles 
décomposaient les éléments pour en identifier les niveaux de signification et en dégager les articulations. Ce courant a également suscité une approche "syntaxique » de l'espace bâti, considéré du point de vue de la matérialité de ses formes et des formes de sa matérialité ${ }^{4}$, en somme une approche structurale se situant au seul plan des signifiants. Il ne s'agit plus dans ce cas d'une sémiotique à proprement parler qui à l'échelle de la ville ${ }^{5}$, doit aussi s'intéresser à la production et à la lecture du sens.

La vague sémiotique des années soixante et soixante-dix a peut-être eu pour effet que l'on méconnaisse d'autres recherches, menées dans le domaine de la linguistique entendue dans un sens non analogique. Des approches diverses ont en commun de s'intéresser à la manière dont le langage ordonne, informe l'espace, ou à la langue en tant qu'elle est « aux prises avec l'espace et le temps ${ }^{6} »$, avec les relations qu'entretiennent mémoire et territoires. La dialectologie, la socio ou l'ethnolinguistique, et la sémantique identifient des champs au sein desquels les mots «jouent » en s'appliquant à des toponymes, à des dénominations, à des catégories, en traitant des matériaux divers, comme des corpus cartographiques $^{7}$ ou des mythes - que l'on pense aux travaux fondateurs de Jean-Pierre Vernant ${ }^{8}$. Elles ne s'intéressent pas seulement aux mots de l'espace, aux dénominations, mais aussi aux façons distinctives de parler dans l'espace ${ }^{9}$ : la ville est constituée de territoires dont les limites sont plus ou moins nettes, plus ou moins perméables ou étanches, stables ou plastiques, où les identités religieuses, culturelles, sociales s'expriment, se révèlent, s'affichent, se trahissent, ou se dissimulent, et interagissent. Les topographies qui se dessinent de la sorte, que l'on cartographie dans des atlas, rendent compte de la diversité linguistique qui se manifeste à l'échelle de groupes larges. Mais cette diversité s'observe également à l'intérieur d'entités plus restreintes, comme une famille, ou encore chez un locuteur qui peut recourir à plusieurs registres en les combinant dans un même énoncé, ou en y faisant appel au gré des situations sociales et spatiales auxquelles il est confronté et selon les positions qu'il occupe ou prétend occuper, en ajustant avec plus ou moins de «talent » ou d'efficacité sa manière de parler. En tentant de conjurer l'imprévu et les risques de malentendu ${ }^{10}$.

5 Il convient d'évoquer aussi les recueils lexicaux qui ont été établis, à partir de traditions orales, de témoignages, de corpus écrits et, plus particulièrement, à partir de la littérature, considérée comme un conservatoire des « virtuosités quotidiennes » dont « la science ne sait que faire et qui deviennent, bien reconnaissables par les lecteurs, les signatures des micro-histoires de tout le monde ${ }^{11} »$. Ils constituent un patrimoine et sauvegardent des parlers menacés, ils mettent au jour des couches de significations oubliées.

Selon une formule de Durkheim, «l'espace ne saurait être lui-même si, tout comme le temps, il n'était divisé et différenciée ${ }^{12}$ ». Qu'il soit qualifié indirectement, par la manière dont on parle dans la ville, ou directement par les mots qui le nomment, le surnomment, le commentent, aux diverses échelles où il est appréhendé par l'analyse ou par l'usage quotidien, l'espace est structuré par des différences pratiques et symboliques. Celles qu'institue le langage relèveraient, dans le vocabulaire durkheimien, des «représentations collectives» ou, dans celui des premières Annales, de l'« outillage mental». Toutefois, si l'on suspend la convention de l'analyse synchronique ou - ce qui revient au même pour ce qui nous intéresse ici - de la "longue durée ", on est conduit à interroger les processus qui produisent et modifient ces formalisations du social. On s'aperçoit alors qu'elles ne sont ni aussi partagées, ni aussi stables qu'on pourrait le croire. 
7 S'agissant d'espaces, d'identités collectives ou d'institutions, lorsque la langue désigne des « objets », en leur conférant du sens elle les constitue. L'assignation du nom distingue et regroupe, ordonne et qualifie. Or, l'accord sur ces points est rien moins qu'assuré. Dans des sociétés différenciées et hiérarchisées de multiples façons, les individus viennent au monde social dotés de schèmes classificatoires différents. Ceux-ci relèvent de l'évidence pratique, de postures et de regards qui s'expriment autant dans des façons de faire que dans des façons de dire. Ils permettent à chacun de donner sens à l'espace social et à sa propre position au sein de celui-ci, mais aussi d'organiser ses conduites au mieux des ressources dont il dispose et de ce qu'il conçoit comme ses intérêts. La reproduction d'un ordre social quelconque tolère fort bien de multiples décalages entre les schèmes classificatoires propres aux différentes positions qui s'y inscrivent, mais elle implique aussi que certains de ces schèmes soient l'objet d'un accord suffisamment général pour faire tenir l'ordre dans le registre symbolique. Ce sont ces systèmes de classement communs qui sont objectivés dans des institutions et dans une langue officielle, seule légitime, qui est, dans les sociétés «modernes », la langue nationale.

8 Depuis une quinzaine d'années, des travaux s'attachent à mettre au jour les « luttes de classement» - pour reprendre le vocabulaire de P. Bourdieu - qui sont au principe de la dynamique et de l'éventuelle intelligibilité des classifications relevant du sens que l'on suppose commun dans une société donnée ${ }^{13}$ : on sait mieux désormais que le langage est vecteur aussi bien qu'indice des conflits et de leurs issues. Ces recherches permettent d'inscrire les systèmes lexicaux et leurs changements dans des processus sociaux où les «représentations» sont constitutives de la "réalité». Elles ne sont plus en effet regardées comme des redoublements objectifs (science), intéressés (idéologie) ou arbitraires (culture) d'un monde social qui serait là «objectivement», mais comme des formes de l'expérience de celui-ci en même temps que des moyens pour le changer ${ }^{14}$. Dès lors, les processus classificatoires peuvent être étudiés dans de multiples dimensions dont il importe d'observer les relations : ils relèvent de la pratique et de ses éléments matériels autant que de la symbolisation langagière, ils impliquent des conflits réglés autant que des consensus sociétaux, ils engagent des mises en ordre institutionnelles autant que des assignations de sens locales et fugitives.

\section{Langues multiples, langue commune}

D'une part une langue " administrante ", dont la visée est organisatrice, d'autre part des façons de parler ordinaires, quotidiennes. Deux polarités auxquelles correspondent deux types de balisage: l'un qui privilégie «les positions relatives des objets les uns par rapport aux autres », et où "le sujet n'intervient pas de façon centrale », l'autre qui au contraire implique, pour le locuteur, une négociation du sens des mots en fonction des situations où il les prononce ${ }^{15}$. Du vocabulaire des institutions et des acteurs, professionnels de l'aménagement, politiques et savants, qui entendent légiférer dans le domaine des mots de la ville, aux lexiques, diversement spécialisés, des différentes catégories de la population, il existe, à un moment donné de l'histoire, au sein d'une même langue, une pluralité de registres, dont les écarts sont des marqueurs de distances sociales et des indices de conflits sur l'assignation du sens.

10 Le point de vue s'enrichit encore si l'on prend en compte la pluralité des langues. L'axiome qui nous guide dans une entreprise collective portant sur plusieurs aires linguistiques est simple à énoncer: chaque langue découpe les réalités, urbaines en 
l'occurrence, de façon spécifique et, par conséquent, un glossaire universel assurant des équivalences terme à terme est un non-sens. Il faut comparer des systèmes et non postuler des correspondances entre éléments. Ce que l'on peut tenir pour une évidence scientifique se heurte, bien entendu, à une autre, pratique : depuis la nuit des temps, le commerce entre groupes linguistiques s'accompagne de traductions. Ce paradoxe classique offre à l'enquête sur « les mots de la ville » de nouveaux objets.

Une remarque ici s'impose, dont nous emprunterons la formulation à Marc Bloch. S'interrogeant sur la traduction et relevant que de nombreuses sociétés connaissent un «bilinguisme hiérarchique », il avance : «Cette opposition de deux langues, forcément différentes, ne figure, en vérité, que le cas limite de contrastes communs à toutes les sociétés. Jusque dans les nations les plus unifiées, comme la nôtre, chaque petite collectivité professionnelle, chaque groupe caractérisé par la culture ou la fortune possède son système d'expression particulier ${ }^{16}$. " En effet, la pluralité des registres de langues dans une même société urbaine où l'on parle en principe une seule langue nationale entraîne des phénomènes analogues, bien que moins apparents, à ceux que l'on observe dans les villes, nombreuses, où existe effectivement un bilinguisme ou un plurilinguisme, ou bien dans les situations de contact entre langues nationales. Dans tous ces cas, les parlers ou les langues doivent communiquer et les solutions à ce problème seront marquées par une négociation inégale entre les locuteurs.

Il n'est donc pas surprenant que l'idée d'un glossaire universel se double généralement de la croyance que, dans chaque aire linguistique, un langage noble, technique ou savant serait le langage de tous ou, du moins, le seul légitime. Elle postule aussi que, dans les relations entre aires linguistiques ou pays, une langue véhiculaire, c'est-à-dire dominante, puisse être une lingua franca. Dans les administrations des Empires et dans les situations coloniales, la langue véhiculaire sera généralement celle de la Puissance centrale ou, du moins, de sa classe dominante. Mais en Europe, dans les rapports entre États-nations, avec l'éclatement des aristocraties francophones et la montée de nouvelles bourgeoisies, la question se pose dans des termes différents : sur fond de concurrence des langues, celle de la nation internationalement dominante tendra à s'imposer soit comme langue véhiculaire obligée, soit comme langue de référence à partir de laquelle seront établies les équivalences. On sait que le français pouvait avoir encore cette prétention au début $d u x^{e}$ siècle et qu'il a été remplacé dans ce rôle par l'anglais américain, au moins depuis la Seconde Guerre mondiale.

Ce qui nous intéresse ici, c'est la quête d'une langue de référence commune aux spécialistes de l'aménagement urbain lorsque se développent et s'institutionnalisent des courants d'échanges internationaux qui imposent de pratiquer couramment la traduction - à la fois «impossible» et nécessaire - ou le bilinguisme. Le phénomène prend sans doute sa forme moderne au $\mathrm{XIX}^{\mathrm{e}}$ siècle. Le commerce et la science sont alors conçus comme porteurs d'une exigence de communication universelle qui se manifeste par l'intensité croissante des relations internationales dans les milieux industriels, scientifiques et réformateurs. À partir de 1850, les expositions « universelles » résolvent le problème à leur manière en adoptant sans émoi la langue du pays organisateur, et la plupart des congrès internationaux font de même. Mais, stimulées par les unes et par les autres, des relations permanentes s'instaurent entre spécialistes de différents domaines de la connaissance et de l'action et les associations internationales se multiplient.

On observe qu'une part importante, parfois essentielle, de leur activité, consiste précisément à établir un langage commun normalisé qui assure l'équivalence des 
catégories permettant de décrire le monde et, du même coup, leur traduction univoque dans les diverses langues de leurs membres. Exemple bien connu, l'Institut international de statistique, créé en 1885, travaille opiniâtrement à définir les cadres uniformes d'une observation statistique universelle autorisant la comparaison ${ }^{17}$. Le même projet est exprimé en 1900 par l'Union internationale des enquêtes économiques et sociales, tentative sans grand lendemain de constituer un « Office international du commerce » à l'image de l'office international du travail de Bâle, qui constituera après la Première Guerre mondiale le noyau du Bureau international du travail. Un des promoteurs de l'initiative constate que, lorsqu'un gouvernement envoie à l'étranger un enquêteur recueillir des renseignements sur des questions économiques et sociales, les difficultés sont encore plus grandes que dans les enquêtes nationales : « Ici, ce n'est plus seulement une transplantation partielle qu'il faut subir: ce sont des argots et des patois nouveaux, des méfiances, des malentendus, des hostilités même déclarées. » Avec l'Union proposée, on se mettra d'accord sur les mots : «Les termes usités sont définis et forment une sorte de vocabulaire international qui détermine les équivalences. » Ensuite, des spécialistes autorisés seront prêts à répondre à toutes les demandes venues de l'étranger. "Le principe : chacun son pays, chacun sa langue, déjoue la plupart des causes de retard et de mécomptes. » L'enquête faite, « le travail de traduction s'opère alors ${ }^{18}$ ».

Cette conviction ancienne restera au fondement des tentatives répétées des organisations intergouvernementales de la Société des Nations, puis de l'Organisation des Nations Unies pour produire, dans leurs différents domaines d'activité, le " vocabulaire international » dont elles estiment avoir besoin. Des situations de même type sont observables dans les institutions de l'Union européenne et dans des pays à bilinguisme officiel. Des enquêtes sur les mots de la ville dans ces différents contextes et, plus largement, sur les traductions de textes administratifs, techniques ou savants permettraient d'identifier les solutions successivement retenues et leurs implications à la fois sur l'échange international et sur les différentes langues.

\section{Approches}

Interroger ainsi les lexiques conduit à faire appel à des méthodes et traditions disciplinaires diverses tout en s'efforçant de réunir les conditions d'une confrontation utile des enquêtes et de leurs résultats.

17 Si ce n'est pas ici le lieu d'exposer les dispositifs pratiques que nous mettons en place pour permettre le développement d'une telle entreprise collective, nous pouvons toutefois indiquer ce qu'il nous est permis d'espérer. Ce qui rend à nos yeux le projet plausible, c'est une conjoncture intellectuelle, relativement synchrone dans plusieurs disciplines des sciences sociales et dans divers pays, qui transforme en objet d'enquête les catégories savantes elles-mêmes et leurs relations avec les catégories communes. Cette attitude renouvelle l'épistémologie des disciplines en faisant l'histoire et la sociologie de leurs institutions, de leurs pratiques et de leurs concepts. Elle désigne à l'attention des chercheurs un vaste domaine d'investigation où ils sont eux-mêmes inclus : les langages organisateurs du social. Elle crée le déplacement infime qui permet désormais à de nombreuses recherches sur les villes de prendre le temps d'être attentives aux langages des acteurs avant de forger, si elles le souhaitent encore, les bons concepts qui leur permettront de dire le vrai. 
18 La question des mots de la description dans les sciences sociales est évidemment ancienne. Pour n'évoquer qu'une tradition proche, M. Bloch s'est longuement interrogé sur le « problème fondamental de la nomenclature » que soulève la pratique de l'historien 19. Celui-ci doit-il « reproduire ou calquer la terminologie du passé » ou bien effectuer son propre «travail de classement» tout en évitant l'anachronisme? Comment peut-il transposer des termes d'une autre langue dans la sienne, « entreprise grosse de périls »? Que faire du vocabulaire des documents alors que ceux-ci ne livrent que le langage des lettrés? De telles questions sont exactement celles que rencontre une recherche sur « les mots de la ville ». Et cependant, l'intention qui leur donne sens n'est plus la même. M. Bloch visait à retrouver la «réalité » à travers et malgré les variations des terminologies d'époque et en dépit des décalages entre « les changements des choses » et " les changements des noms ». Il cherchait du même coup à produire des nomenclatures scientifiques qui décrivent les choses sans les trahir. Notre propos est différent : il est de prendre les classements des acteurs, dans toute leur variété, comme un aspect de la "réalité » elle-même. Nous pouvons ainsi faire l'économie de la question d'un « référentiel » qui devrait être énoncé dans d'autres mots que ceux que nous recueillons.

Il s'agit donc de constituer des corpus qui permettent de restituer, pour des groupes relativement homogènes de locuteurs et dans des points particuliers du temps et de l'espace, des usages. Il s'agit aussi d'observer les migrations de termes d'un groupe de locuteurs à l'autre, les changements de sens ou de forme qui les accompagnent et, ainsi, la formation et les changements de la langue commune. Il s'agit, enfin, de repérer les systèmes classificatoires qui organisent à chaque moment les lexiques, qui se confrontent et qui se transforment. L'enquête porte d'abord sur deux champs sémantiques limités : d'une part, les lexiques qui appréhendent la ville en tant qu'entité (désignations génériques ou classificatoires des villes), d'autre part, ceux qui nomment les territoires qui composent la ville (en particulier les unités prenant place entre la sphère domestique et la ville dans son ensemble).

L'entreprise n'est pas trop difficile, bien que terriblement laborieuse, pour ce qui est des lexiques administratifs, techniques ou savants. Ceux-ci sont importants pour notre projet car, d'une façon ou d'une autre, ils vont s'inscrire dans des institutions et des espaces bâtis qui constituent le cadre de la vie quotidienne des populations. Les citadins, dans leur diversité sociale, seront donc confrontés à une terminologie officielle qu'ils pourront adopter ou refuser, le plus souvent transformer ou détourner. Les migrations du lexique administratif dans les parlers communs sont constantes et réservent bien des surprises. Du jargon de la loi foncière française de 1957 qui instituait les «zones à urbaniser en priorité » au «tu habites la zup ? » d'aujourd'hui, du lexique des fonctionnaires de la Works Project Administration du New Deal américain au «this is not a project » de ceux qui, un demi-siècle plus tard, ne veulent surtout pas apparaître comme habitant un logement municipal, sont intervenus à la fois un emprunt et un changement de sens. Un cas méritant attention, en français, est celui des mots «zone ", "zoner ", " zonard", transformations récentes de « la zone » et « zonier » qui, eux-mêmes, réinterprétaient la «zone non aedificandi » instaurée en 1841 en même temps que la nouvelle enceinte de Paris. D'autres vocables bureaucratiques, en revanche, ne se sont pas imposés et restent donc cantonnés dans les sphères qui les ont produits : les boros qui divisent New York n'ont sans doute que des noms propres pour les habitants, les «districts » ou « communautés urbaines » françaises sont probablement des notions exotiques pour ceux qui y vivent. 
21 La circulation de vocabulaire entre le monde politico-administratif et celui des techniciens et savants - ingénieurs, statisticiens, géographes ou sociologues, notamment - est aussi un phénomène à explorer car, non seulement il contribue à façonner les mots de la ville, mais il est aussi un révélateur des rapports entre ces ordres de pratiques. Dans quelles circonstances des catégories d'origine purement administrative se solidifient en « concepts » dans les disciplines des sciences sociales? À l'inverse, par quels processus le monde savant fournit-il parfois le langage qui va permettre au monde politique et aux faiseurs d'opinion de mettre en forme des situations de trouble de l'ordre social comme des «problèmes urbains »? «The inner-city problem » aux États-Unis dans les années cinquante et 1960 et ses transformations ultérieures, «le problème des banlieues » en France dans les années quatre-vingt et quatre-vingt-dix peuvent ainsi être étudiés comme ce qu'ils sont d'abord: des faits de langage.

Les registres de langue que l'on vient d'évoquer sont accessibles dans des corpus écrits, généralement imprimés, qui posent surtout le problème de leur extrême abondance. En revanche les parlers communs requièrent des enquêtes spécifiques, où l'écoute et l'enregistrement en situation sont privilégiés. Pour exploiter les corpus ainsi constitués, il est nécessaire de les soumettre à des opérations de transcription qui restituent, non seulement la syntaxe, mais éventuellement les caractères prosodiques de la parole recueillie, et dont l'oralité doit être sauvegardée dans le passage à l'écrit. Lorsqu'il y a diglossie, il arrive que le chercheur rencontre un obstacle qui n'est pas d'ordre méthodologique. Il tient à une conception selon laquelle le parler n'a pas la légitimité d'une langue et ne saurait être écrit, seule la langue légitime - voire sacrée, lorsqu'elle est celle de la Révélation - étant digne de l'être.

23 À l'aide de ces diverses sources orales et écrites, il est possible de produire divers résultats. Mentionnons seulement deux des genres que l'on peut retenir pour la conduite de l'enquête et la restitution de ses trouvailles. L'un est ce que nous appellerions volontiers "les aventures de mots ", récits qui s'efforcent de reconstituer, en voyageant entre divers univers de locuteurs pendant des périodes suffisamment longues, l'émergence d'un mot, ses changements d'acception et d'usage, les concurrents qu'il rencontre, transforme, élimine ou, à l'inverse, qui entraînent sa disparition. L'exercice est classique et L. Febvre, qui s'y est essayé, nous rassure : « Faire l'histoire d'un mot, ce n'est jamais perdre sa peine ${ }^{20}$.» M. Bloch invite aussi à pratiquer cette "sémantique historique » dont il trouve d'« admirables modèles » chez Fustel de Coulanges, et précise : "L'avènement du nom est toujours un grand fait en histoire ${ }^{21}$. " Bien entendu, les risques de l'entreprise sont multiples, notamment négliger les systèmes dans lesquels le terme qui nous intéresse prend sens, ne pas apercevoir des analogues ou concurrents importants, ignorer les usages qui n'ont pas laissé de traces écrites. Mais les profits ne sont pas moindres: les aventures de mots permettent de travailler d'emblée sur les interactions entre registres et de repérer les dates et les lieux des innovations et, de cette façon, les contextes sociaux dans lesquels le langage se transforme.

Un autre genre tout aussi classique, mais plutôt pour les linguistes que pour les historiens, est l'analyse synchronique des systèmes dans un champ lexical défini, qui s'ouvre ensuite sur la comparaison entre systèmes contemporains ou successifs. L'enquête est ici très différente de la précédente : la recherche s'attachant exclusivement aux relations entre éléments, l'homogénéité et la simultanéité du corpus sont fondamentales. Une telle méthode est particulièrement pertinente lorsque les occurrences rencontrées prennent ou se voient donner la forme de définitions qui 
présentent les propriétés logiques d'une classification. Les corpus de nature juridique ou les traités d'administration se prêtent aisément à de telles analyses. Il en est de même des dictionnaires de langue, spécialement ceux dont les auteurs ont adopté la philosophie de la "définition parfaite», c'est-à-dire par le genre et les traits spécifiques ${ }^{22}$. Voyons quelques adresses dans celui de Furetière, publié en $1690^{23}$ :

«BOURG. Habitation de peuple qui tient le milieu entre la ville et le village. Quelques-uns le restraignent aux lieux qui ne font fermez ni de murs, ni de fossez.

$[\ldots] »$

«Faus-bourg. Habitation de peuple attenant les portes d'une ville. [...] »

«Bourgade. Diminutif de bourg.»

«CITÉ. Ville fermée de murs. [...] Ce mot ne se dit proprement que des villes épiscopales. [...]»

«Quartier, signifie aussi un certain canton ou division d'une ville.»

«VILLAGE. Habitation de paysans qui n'est point fermée de murs, \& qui a d'ordinaire une Paroisse. [...]»

« VILLE. Habitation d'un peuple assez nombreux, qui est ordinairement fermée de murailles. [...] Ville signifie quelquefois une partie d'une grande ville. La vieille ville, la ville neuve. À Paris il y a Ville, Cité \& Université. »

Pour les mots retenus ici - aux seules fins d'illustration - deux définisseurs emboîtés («habitation de peuple» et «ville») et un petit nombre de marques spécifiques (le nombre, les murs, l'évêché, la paroisse, la division) déterminent un système qui fait de " ville ", «bourg ", "faus-bourg » et "village » des espèces du même genre, comme par ailleurs « ville » (dans une seconde acception) et « quartier », tandis que « cité » apparaitt comme une modalité de "ville». Cette hiérarchisation insolite des catégories spatiales, repérable en France à la veille d'une "crise des définitions urbaines ${ }^{24}$ », appellerait une comparaison avec celle que l'on observe dans d'autres dictionnaires ou documents analogues pour d'autres langues ou d'autres périodes.

\section{Situations}

Si l'on regarde les mots de la ville comme indices des dynamiques des sociétés urbaines qui les créent et les emploient, il convient d'être particulièrement attentif à certaines situations susceptibles de nourrir la réflexion comparative.

À cet égard, l'étude de cas de réforme est éclairante. Admettons d'emblée l'ambiguïté du terme: la réforme de la ville et celle de ses mots sont intimement liées. Depuis les premiers énoncés d'une "question urbaine » au temps des Lumières, différents corps de spécialistes ont continûment dessiné, en Europe et en Amérique du Nord, les figures successives d'un projet "modernisateur » ou " rationalisateur » sur la ville et la société. Ils ont inventé des instruments d'observation et de mesure, de diagnostic et de prescription, dont la condition de possibilité a été la création de langages. D'abord savants, confinés aux milieux réformateurs et techniciens, ils s'inscriront peu à peu dans de nouveaux dispositifs administratifs et statistiques, et, finalement, dans des espaces où vivront les citadins.

La réforme des mots de la ville transforme les objets qu'ils sont censés nommer. On pourrait sans doute écrire l'histoire du mot slum dans cette perspective ${ }^{25}$. Terme de l'argot populaire de Londres (cant), il désigne d'abord « a room of low repute » ou « low, unfrequented parts of the town ». Pendant la majeure partie du XIX siècle, il apparaît par écrit plutôt sous la forme back-slum(s) et, en règle générale, entre guillemets. La montée 
$\mathrm{du}$ mouvement pour la réforme du logement conduit alors à une série de transformations: le terme reçoit une acception technico-juridique, pour désigner «a house materially unfit for human habitation » et slums passe dans le langage courant, perdant ses guillemets à l'écrit dans les années 1880 , au même moment où suburbs ou suburbia émergent comme catégories de la diversité des noms de lieu. D'un terme vulgaire, stigmatisant et rare, à l'objet indécis, la réforme a fait un concept général et opératoire : il est possible désormais de délimiter sur une carte des slum areas. L'histoire continuera au $\mathrm{xx}^{\mathrm{e}}$ siècle, de part et d'autre de l'Atlantique, marquée par deux mouvements liés qui aboutiront à la désuétude du terme. D’un côté, l'adoption de législations autorisant la démolition imposera des définitions rigoureuses qui ne peuvent se satisfaire du halo de connotations qui accompagnait slums et aboutiront à l'adoption de nouveaux termes «techniques» qui permettront du même coup d'euphémiser la stigmatisation sociale des habitants : aux États-Unis, tenement-house, tenement district, puis deteriorated neighbourhood apparaîtront entre les années 1890 et les années trente. Parallèlement, un nouveau regard sur les quartiers populaires apparaît avec l'émergence $\mathrm{du}$ «travail social » à partir des années dix : dans ces contextes, les slums de la fin du XIX siècle pourront être élevés à la dignité de neighbourhoods ou communities par la grâce d'un projet de reconstruction des relations sociales. Ce changement rend sans doute possible l'adoption par les city planners américains des années vingt du concept de neighbourhood unit qui va s'incorporer pour longtemps au vocabulaire international de l'urbanisme.

L'aventure de favela peut aussi être évoquée ${ }^{26}$. C'est d'abord un nom propre : peu après que les habitants des quartiers de Rio de Janeiro victimes de "l'ère des démolitions » eurent commencé à envahir de leurs « cases » (casebres) le Morro da Providencia, une des collines du centre-ville, l'usage populaire rebaptise celle-ci, vers 1897, « Morro da Favela ${ }^{27}$ ». Un nouveau type d'habitat de fortune est apparu, distinct par sa morphologie et son illégalité des habitations collectives dégradées du tissu ancien, désignées par estalagens et cortiços. Pour les autorités comme pour la presse, «la Favela » est d'emblée un (anti)modèle, associé au crime et au désordre, auquel seront de plus en plus souvent comparés d'autres sites. Des constructions similaires se multiplient en effet sur les escarpements de la ville, mais il faudra attendre les années vingt pour que le toponyme soit substantivé : favela perd alors sa majuscule, puis les guillemets d'abord utilisés, pour devenir un nom commun. Favelas donne alors naissance à favelados, une population étant ainsi désignée par son habitat. La nouvelle catégorie est adoptée par l'administration municipale lorsque, avec l'Estado Novo, une ambitieuse politique d'éradication devient envisageable : dans les années quarante sont réalisés les premiers « recensements des favelas » et cellesci existent désormais de jure. Le terme sera par la suite communément utilisé dans d'autres villes, comme São Paulo, dont la topographie est pourtant toute différente.

Avec le mot arabe dâhiya on a l'exemple d'une trajectoire inverse puisque, de nom commun, il est devenu à Beyrouth un quasi-toponyme. Hérité de la langue classique, et ressortissant à un registre politico-administratif, dâhiya (employé souvent au pluriel, dawâhî) signifie notamment "environs, abords, périphérie, banlieue ». Il semble s'être intégré dans les dialectes orientaux assez récemment: dans les années trente le dictionnaire de Barthélémy, tenu pour une autorité en la matière, ne mentionne pas encore cette acception, qui figurera dans son supplément publié en $1960^{28}$. Au Liban, au cours de la guerre civile, il en vient à désigner couramment et exclusivement la banlieue sud de la capitale, c'est-à-dire la banlieue chiite, assimilée à un espace pauvre, anarchique, illégal et intégriste. D'où le refus de certains de ses habitants d'utiliser ce 
terme et la volonté de revenir plutôt aux anciens toponymes, ou de recourir à une désignation administrative moins connotée, comme « le littoral de Metn sud ${ }^{29}$ ».

Slum, favela ou dâhiya relèvent d'un vaste champ sémantique, celui de la stigmatisation urbaine, qui se prête sans doute bien à la réflexion comparative. En effet, des ressources lexicales variables selon les langues sont travaillées ici par des processus sociaux et sous des contraintes sémantiques semblables. L'émergence d'une catégorie qui, dans le cas de slum et de favela, subsume la diversité des toponymes n'est-elle pas liée à une opération cognitive de diagnostic elle-même subordonnée à des objectifs de réforme? De simples tableaux chronologiques peuvent être sur ce point très éclairants. On observe, à divers moments de l'histoire, que plusieurs vocables sont susceptibles de fournir la catégorisation dont la réforme a besoin: leur concurrence n'est-elle pas réglée par des contraintes générales qui conduisent à des solutions diverses selon les contextes d'énonciation et, bien entendu, les ressources lexicales de chaque langue? Ainsi, les mots de la stigmatisation urbaine ont pour propriété commune d'exprimer sur le registre de l'habitat et de l'espace une stigmatisation sociale qui repose, en réalité ou en même temps, sur d'autres critères de hiérarchisation qu'ils font passer au second plan. Comment s'effectue, dans l'un et l'autre sens, le passage de la catégorie spatiale à la catégorie sociale? Ils doivent, en outre, lorsque le projet réformateur s'affirme, être suffisamment généraux pour ne pas renvoyer à un type morphologique trop particulier, tout en restant suffisamment concrets pour rester proches des images et connotations qui les rendent intelligibles. Ils doivent, enfin, lorsqu'ils se trouvent inclus dans des dispositifs réglementaires, statistiques ou opérationnels, être susceptibles de définitions juridiques ou techniques univoques qui fassent disparaître le halo de leurs connotations. Ainsi, les mots de la stigmatisation urbaine peuvent être brutalement péjoratifs dans certains contextes, tandis que, dans d'autres, il est essentiel qu'ils soient massivement euphémisés : émergent alors les lexiques techniques du sub-standard housing ou du social work, qui vont d'ailleurs jeter un pont entre le vocabulaire de la dénonciation et celui, plus large, de leur pratique ou discipline.

Un des traits qui apparaissent dans ces exemples est sans doute commun aux processus de réforme : c'est la création de catégories là où prévalait la diversité des noms de lieux ou des notions descriptives, c'est, en d'autres termes, l'instauration de nouvelles échelles de description, de classement et d'intervention. Il est probable que résulte d'un processus de ce genre le mot « agglomération » qui vient tardivement décrire une réalité physique et spatiale déconnectée des implications juridiques, administratives et politiques de «ville» ou bien, plus tard, metropolitan area qui, parti des États-Unis, fera le tour du monde. Dans de telles réorganisations, certaines disciplines, avec leur tradition lexicale propre, sont plus que d'autres mises à contribution. Ainsi la géographie, périodiquement confrontée à des exigences terminologiques (distinguer, généraliser, formaliser...), propose-t-elle de fixer des significations ${ }^{30}$. Elle réorganise l'espace géographique en recourant à des langages techniques, à des parlers régionaux, en empruntant à des langues étrangères, ou en légitimant des emprunts déjà effectués. C'est ce qu'a mis plus particulièrement en lumière, pour la géographie française, $\mathrm{E}$ de Dainville, en soulignant que celle-ci, «à l'encontre d'autres sciences, [...] dès le XVI ${ }^{e}$ siècle, commence à parler la langue maternelle ${ }^{31} »$.

Dans certains cas, ce qui s'offre à l'observation, c'est une réforme globale de la langue liée à un projet national : qu'il s'agisse de la réforme de l'arabe impulsée au Liban dans la seconde moitié $\mathrm{du}$ XIX ${ }^{\mathrm{e}}$ siècle donnant naissance à la première encyclopédie 
contemporaine dans cette langue, de celle du turc après la fondation de l'État kémaliste ou de celle du chinois par la nouvelle République de Sun-Yat-Sen, nous avons affaire à des interventions volontaristes et coordonnées dont l'État prend l'initiative ou auxquelles il accorde son parrainage. Dans de telles conjonctures, les lexiques urbains sont bouleversés avec tout le reste, mais les solutions retenues ont été préparées et ont fait l'objet de débats spécifiques dans des académies de langue ou des sociétés savantes, dans des administrations ou des organisations professionnelles spécialisées. La réforme des mots de la ville est ici mise au service à la fois d'une affirmation identitaire nationale et d'une volonté de faire entrer les villes dans la modernité : situations quasi expérimentales pour notre recherche.

Il est aussi des stratégies moins globales, plus progressives, qui répondent à une semblable exigence: "naturaliser» des notions empruntées en les intégrant dans la langue indigène, et en même temps marquer leur nouveauté par une innovation langagière - conforme cependant au génie de la langue. C'est ce qu'illustre une "aventure» empruntée au monde arabe. Il ne s'agit pas en l'occurrence d'une dénomination de nouveaux «objets » urbains, ou de nouveaux découpages, au moyen d'un vocabulaire disponible comme dans le cas des thumn-s, «huitièmes ", désignant de nouvelles entités administratives qui prévaudront au Caire à la suite de l'expédition de Bonaparte et dont l'usage oubliera, de la même manière que pour le mot «quartier » en français, sa valeur de fraction - on comptera d'ailleurs après quelques années plus de huit " huitièmes ». Il ne s'agit pas non plus de traductions, de décalques, de transferts d'une langue étrangère exportatrice de modèles, mais d'une "invention" qui puise plus directement dans les ressources de la langue indigène.

Le mot qui prévaut jusqu'au XIX ${ }^{e}$ siècle pour désigner dans le monde arabe un hôpital est un terme d'origine persane, bimaristan ou sa déformation maristan (qui progressivement semble plutôt concerner les lieux où l'on traite les fous). Dans la seconde moitié de ce même siècle apparaît un mot, qui se dit et s'écrit isbitalia ou usbitalia (voire sbidal, selon des archives beyrouthines ${ }^{32}$ ), dont on reconnait aisément l'origine italienne ${ }^{33}$. Il semble alors courant dans l'Empire ottoman, et son emploi est attesté en Turquie jusqu'en 1945. Dans les dictionnaires de turc où il figure (avec un $p$, ispitalia) à la fin du XIX siècle, on précise qu'il désigne surtout (ou qu'il a désigné à l'origine) des établissements, appartenant notamment aux communautés grecques et arméniennes, qui combinent les fonctions d'hospice et d'hôpital ${ }^{34}$. Ensuite l'emploi d'un mot arabe forgé par les académies de langue au tournant du siècle s'est généralisé pour désigner, dans la langue administrative, ainsi que dans le parler quotidien du Machrek, les hôpitaux, selon une acception contemporaine : mustachfâ, «le lieu où l'on cherche la guérison ». Ce terme l'a apparemment emporté non seulement sur la formule plus ancienne dâr al-chifâ, « maison de la guérison ", mais sur celui de machfâ, lui aussi attesté, qui est formé à partir du même radical, mais signifie "lieu de la guérison". Au Caire, si l'on en juge d'après les indications figurant sur le plan détaillé de la ville que l'administration a établi à partir de 1909, le mot isbitalia a été conservé dans l'appellation des voies ou des places (du type: " rue de l'Hôpital français »), tandis qu'il était remplacé par mustachfâ dans la désignation des bâtiments qui leur avaient donné leur nom. Et il n'a pas totalement disparu de la langue parlée ${ }^{35}$. Au Maghreb, la forme sbital, ou sbitar (le $r$ remplaçant le $l$ selon un mécanisme courant), est d'un usage banal.

Ainsi a-t-on procédé à une double "nationalisation" ou arabisation. D'une part pour désigner un équipement «moderne » (à l'occidentale), après avoir emprunté un mot 
européen à titre transitoire, on l'a abandonné, dans la langue écrite du moins. D'autre part on n'est pas revenu au terme ancien, un terme certes persan, mais dont l'usage dans les pays arabes remonte au Moyen Âge. Ispitalia, même une fois le $p$ devenu $b$, ne sonne pas encore vraiment arabe et ce n'est que devenu, après métathèse, istibalia, correspondant en Égypte à un registre très populaire ${ }^{36}$, qu'il épouse un schème morphologique parfaitement classique. Ce dernier ajustement n'a pas cependant de fondement sémantique et l'arabisation est dans ce cas uniquement formelle. Avec mustachfâ, la réforme a, elle, produit un mot nouveau " authentiquement » arabe, que l'usage social a consacré, et elle l'a fait en opérant un choix sémantique extrêmement précis et motivé, tout en appliquant un procédé permettant d'obtenir, de façon "générative» par dérivation à partir d'un radical, diverses formes verbales, des substantifs, et en particulier des noms de lieu.

À plusieurs égards une telle aventure, qui en l'occurrence n'est pas celle d'un seul mot, a une valeur exemplaire. À ce titre elle pourrait être rapprochée, et par certains de ses aspects distinguée, d'autres trajectoires. Sans doute les situations de réforme - et cela est vrai pour toute transformation délibérée ou non du langage - nous rappellent-elles d'abord qu'une langue a une capacité, morphologique et phonologique, qui lui est spécifique pour intégrer de la nouveauté ou en inventer. Et pour opposer une résistance, ne serait-ce que par les déformations auxquelles elle soumet, en se les appropriant, des éléments de systèmes qui lui sont exogènes : il n'y a pas de transfert sans modification. Block devient égyptien lorsqu'il se prononce bulukk, et «hangar », quand au pluriel il se plie à un schème arabe, hanâger.

Plus encore, ces situations attirent notre attention sur la manière dont des choix sont effectués, abandonnés, adoptés, intégrés dans l'usage, sur les moyens mis en œuvre pour les appliquer ou les infléchir, sur les raisons des succès ou des échecs et les enseignements qu'il est possible d'en tirer. Et la comparaison permet de dégager ce que certains processus ont de singulier ou au contraire de « comparable ».

Les résistances ne tiennent pas toujours et seulement à une inertie qui renvoie à la structure de la langue. Elles sont également d'ordre sémantique. Par exemple il y a quelques années, pour «baptiser » de nouveaux ensembles de logements construits à la périphérie du Caire, les urbanistes égyptiens, jugeant inadéquat le vocabulaire usuel dont ils disposaient, ont suggéré l'emploi d'un équivalent du terme anglais neighbourhood. Pour cela ils ont utilisé, comme cela avait été fait pour mustachfâ les ressources morphologiques de l'arabe. Or le mot retenu, mugâwara, a été rejeté par les habitants pour lesquels il avait une connotation négative. En effet si ce terme peut évoquer la proximité des lieux saints de l'Islam, il était en l'occurrence associé à l'habitation traditionnelle des étudiants pauvres (dits mugâwarîn, vivant au voisinage ou dans les dépendances de la mosquée-université d'al-Azhar et des lieux d'enseignement) et, par extension, à l'occupation des cimetières à des fins de résidence (une vie au voisinage des tombes) ${ }^{37}$. En définitive ils ont adopté ou, plutôt, "réactivé » un terme populaire (et ancien: il n'appartient pas au vocabulaire de la partie moderne de la ville) : hâra. Les aménageurs croyaient bien faire en rendant dans leur langue une notion venue d'ailleurs, convaincus qu'ils étaient de son caractère positif (et cela d'autant plus qu'en arabe l'idée de voisinage est « naturellement » associée à celles d'hospitalité et de protection). Les habitants, quant à eux, n'ont « entendu » qu'un mot stigmatisant.

On pense inévitablement à d'autres termes qui ont pris dans le vocabulaire journalistique et politique français d'aujourd'hui la force d'évidences. Nous relevions plus haut que 
l'émergence du « problème des banlieues » depuis une dizaine d'années apparaît d'abord comme un fait de langue. Dans la longue série des mots de la ville qui ont pour fonction de désigner les territoires de la stigmatisation sociale et d'énoncer un état de la "question urbaine ", il s'agit d'une innovation. Il ne serait pas inutile de localiser les origines de cet usage dans l'espace social, d'examiner comment il est repris par certains locuteurs et contesté par d'autres, bref de l'analyser comme l'indice d'une (très vive) lutte de classement. D'autres termes, notons-le, sont en concurrence, dont l'histoire est toute autre : «cités » emprunte aux « cités ouvrières » de la fin du XIX ${ }^{e}$ siècle, mais aussi au vocabulaire historisant d'un Marcel Poëte (qui a lu Fustel de Coulanges) et, plus généralement, des urbanistes des années 1910 et 1920 ; « les quartiers », dans certaines villes françaises, dit autrement la même chose. Et comment parlera-t-on désormais des «banlieues résidentielles » vantées par les promoteurs immobiliers des années soixante et soixante-dix ? Voilà un argument, peut-être, pour passer des excursions étymologiques sur un mot qui nous soucie à un travail plus méthodique sur les systèmes classificatoires inscrits dans le langage.

\section{NOTES}

1. Piloté par le PIR-Villes du CNRS et le programme MOST de l'Unesco, le programme «Les mots de la ville » s'attache aux registres de dénomination de la ville et de ses territoires en usage dans différentes aires linguistiques. Il privilégie le comparatisme, à l'intérieur d'une même aire et d'une aire à l'autre, et il prend en compte la longue durée.

2. R. Barthes, « Sémiologie et urbanisme", in L'aventure sémiologique, Paris, Seuil, 1985, p. 261. Cf. également La Tour Eiffel, Paris, Delpire, 1969.

3. On trouvera des réflexions sur l'état du champ, qui incluent un retour sur des recherches plus anciennes, in S. Ostrowetsky, ed., Sociologues en ville, Paris, L'Harmattan, 1996, troisième partie, « La ville en langue ».

4. Cf. notamment les travaux de J. Castex et P. Panerai (en particulier in Sémiotique de l'espace, Paris, Gonthier, 1979).

5. À l'échelle de la ville, R. Ledrut a souligné la difficulté d'une entreprise sémiologique (Les images de la ville, Paris, Anthropos, 1973).

6. La formule est de E. Noël, in E. Noël et G. Minot, eds, L'espace et le temps aujourd'hui, Paris, Seuil, 1983, p. 223.

7. F. de Dainville, Le langage des géographes, Paris, Picard, 1964.

8. Mythe et pensée chez les Grecs, Paris, Maspero, 1965.

9. Cf. la synthèse de L.-J. Calvet, Les voix de la ville. Introduction à la sociolinguistique urbaine, Paris, Payot, 1994.

10. À propos de la notion de malentendu, cf. plus particulièrement J. Gumperz, Engager la conversation. Introduction à la sociolinguistique interactionnelle, Paris, Minuit, 1989.

11. M. de Certeau, L'invention du quotidien, Paris, UGE, 1980, vol. 1, Arts de faire, p. 138.

12. É. Durkheim, Les formes élémentaires de la vie religieuse [1912], Paris, Presses universitaires de France, 1960, p. 15.

13. Évoquons seulement, dans cette orientation de recherche: L. Boltanski, Les cadres. La formation d'un groupe social, Paris, Minuit, 1982 ; A. Desrosières et L. Thévenot, Les catégories socio- 
professionnelles, Paris, La Découverte, 1988 ; J. W. Scott, Gender and the Politics of History, New York, Columbia University Press, 1988 ; C. Charles, Naissance des « intellectuels», 1880-1900, Paris, Minuit, 1990 ; C. Topalov, Naissance du chômeur, 1880-1910, Paris, Albin Michel, 1994 ; G. Noiriel, « Sociohistoire d'un concept : les usages du mot "nationalité" au XIX ${ }^{e}$ siècle », Genèses, 20, 1995, p. 4-23.

14. De telles propositions furent notamment au principe d'un renouveau de l'histoire urbaine en France. Cf.

J.-C. Perrot, Genèse d'une ville moderne. Caen au XVIII siècle, Paris-La Haye, Mouton, 1975, 2 vol., et B. Lepetit, Les villes dans la France moderne (1740-1840), Paris, Albin Michel, 1988.

15. Nous empruntons cette distinction à J.-C. Milner, in E. Noël et G. Minot, eds, L'espace et le temps aujourd'hui, op. cit., p. 224-225.

16. M. Bloch, Apologie pour l'histoire ou le métier d'historien [1949], Paris, Armand Colin, 1993, p. 171.

17. Cf. E. Brian, "Y a-t-il un objet "Congrès"? Le cas du Congrès international de statistique (1853-1876) ", Mil neuf cent, 7, 1989, p. 9-22, et A. Desrosières, La politique des grands nombres. Histoire de la raison statistique, Paris, La Découverte, 1993.

18. $\mathrm{P}$. Du Maroussem, "L'union internationale des enquêtes économiques et sociales ", Revue d'Économie politique, XIV, 1900, p. 640.

19. M. Bloch, Apologie pour l'histoire ou le métier d'historien, op. cit., p. 167, 168, 170 et 168.

20. L. Febvre, "Civilisation. Évolution d'un mot et d'un groupe d'idées ", in Première Semaine internationale de synthèse, Civilisation, le mot et l'idée, Paris, Renaissance du livre, 1930, p. 9, rééd. in Pour une histoire à part entière, Paris, SEVPEN, 1962, p. 481. Une autre mise en œuvre : « Travail : évolution d'un mot et d'une idée ", Journal de psychologie normale et pathologique, 1948, p. 19-28, rééd. in Pour une histoire à part entière, ibid, p. 649-658.

21. II poursuit, dans une direction qui n'est pas la nôtre : « même si la chose avait précédé ; car il marque l'étape décisive de la prise de conscience » (M. Bloch, op. cit., p. 174).

22. Sur les définitions des dictionnaires, cf. B. Quemada, Les dictionnaires du français moderne, 1539-1863. Étude sur leur histoire, leurs types et leurs méthodes, Paris, Didier, 1967, p. 391-464.

23. A. Furetière, Dictionnaire universel, contenant généralement tous les mots français tant vieux que modernes,.., La Haye-Rotterdam, A. et R. Leers, 1690, 3 vol.

24. J.-C. Perrot, Genèse d'une ville moderne..., p. 28-51.

25. H. J. Dyos et D. A. Reeder en ont fait l'esquisse en suivant les pistes offertes par l'Oxford English Dictionary, Cf. «Slums and Suburbs », in H. J. Dyos et M. Wolff, eds., The Victorian City. Images and Realities, Londres, Routledge and Kegan Paul, 1973, vol. 2, Shapes on the Ground. A Change of Accent, p. 359-386.

26. Cf. M. de Almeida Abreu, « Reconstruire une histoire oubliée. Origine et expansion initiale des favelas de Rio de Janeiro », Genèses, 16, 1994, p. 45-68.

27. Le mot désigne un arbuste du sertão nordestin et, en particulier, la région de Canudos où viennent de faire campagne les militaires qui obtiennent en 1897 l'autorisation de s'installer sur les pentes du Morro da Providencia.

28. A. Barthélémy, Dictionnaire arabe-français des dialectes de Syrie: Alep, Damas, Liban, Jérusalem, Paris, Paul Geuthner, 1935-1950. C. Denizeau, Dictionnaire des parlers arabes de Syrie, Liban et Palestine (supplément au dictionnaire arabe-français de A. Barthélémy), Paris, Maisonneuve, 1960.

29. Nous devons ces dernières précisions à Mona Harb-El-Kak qui mène des recherches sur cette région (cf. Maîtrise de l'espace dans la banlieue sud de Beyrouth. Recompositions territoriales entre pouvoirs publics et intervenants privés, rapport de DEA, Tours, Université de Tours, 1996, multig.).

30. On trouve une manifestation récente de cette préoccupation dans R. Brunet, ed., Les Mots de la géographie, Paris, La Documentation française, 1992.

31. F. de Dainville, Le langage des géographes, op. cit., p. 319.

32. Cf. M. Davie et L. Nordiguian, "L'habitat de Bayrût al-Qadîma ", Berytus, Arcbeological studies, vol. 25, 1987.

33. Cf. notamment F. Baker, "Questions de toponymie », Égypte/Monde arabe, 5, 1991. 
34. Nous devons ces précisions lexicologiques à A. Le Gall-Kazazian.

35. J.-L. Arnaud, Toponymie du Caire, Le Caire, CEDEJ-MRC, 1994, p. XIII et 245 sq.

36. El-Saïd Badawi et M. Hinds, A Dictionary of Egyptian Arabic, Beyrouth, Librairie du Liban, 1986, p. 18.

37. Mugâwarîn fait partie du langage officiel : c'est la dénomination d'une entité administrative, incluant une grande partie des cimetières de l'est et al-Azhar.

\section{RÉSUMÉS}

Cet article prend appui, en en développant les principales orientations, sur un programme consacré aux « mots de la ville» (la ville en tant qu'entité et les territoires qui la composent). Il s'agit d'identifier, dans différentes aires linguistiques, langues «administrantes » d'une part, façons de parler ordinaires d'autre part, et leurs relations, en observant notamment les migrations de termes d'un groupe de locuteurs à l'autre, les changements de sens ou de forme qui les accompagnent et, ainsi, la constitution et les changements de la langue commune. Et de repérer les systèmes classificatoires qui organisent les lexiques, qui se confrontent et qui se transforment. Il est possible de suivre, à travers le temps, des "aventures de mots ", et de comparer des systèmes contemporains ou successifs à partir de corpus homogènes. Parmi les situations qui nourrissent la réflexion comparative, en permettant de dégager ce que certains processus ont de singulier ou au contraire de commun, les cas de réforme (réforme de la ville et celle de ses mots) sont particulièrement éclairants.

This article develops the main orientations of a programme devoted to the "words of the City" (the City as an entity and the territories that constitute it). It is concerned on the one hand, with identifying languages of administration in the different linguistic spaces and on the other, with ordinary ways of talking and their relationship. It particularly observes migrations of terms from one group of speakers to another, the changes of meaning or form which accompany them and thus the constitution and transformation of the common language. It is also concerned with identifying classificatory systems which organise the vocabularies, confront each other and are transformed. It is possible through time to trace the adventure of words and compare contemporary or successive systems from homogeneous corpuses. Among the situations which foster comparative reflection while allowing us to separate that which is particular to certain processes or on the contrary which is common, the cases of reform (reform of the City and of its words) are particularly illuminating.

\section{AUTEURS}

\section{JEAN-CHARLES DEPAULE}

Jean-Charles Depaule (CNRS, Aix-en-Provence) étudie l'espace habité des villes du monde arabe contemporain. Il est le coordinateur scientifique du programme comparatif « Les mots de la ville » (PIR-Villes-CNRS/MOST-UNESCO). Il a récemment publié avec J. Castex et J.-L. Cohen, Histoire urbaine, anthropologie de l'espace, Paris, Editions du CNRS, 1996. 


\section{CHRISTIAN TOPALOV}

Christian Topalov (CNRS-EHESS) est sociologue des cultures et des sociétés urbaines. Il a notamment publié Naissance du chômeur, 1880-1910, Paris, Albin Michel, 1994. 